FORMATION Formation emploi

Revue française de sciences sociales

98 | avril-juin 2007

Pour une approche par les capacités

\title{
Quel espace de choix des métiers ? Les inégalités d'accès aux savoirs techniques en Guinée Maritime
}

How wide is the choice of occupations? Inequalities in access to technical knowhow in coastal Guinea

Raum für freie Berufswahl? Ungleichheiten beim Zugang zu technischem Wissen in der Republik Guinea

Jean-Étienne Bidou et Isabelle Droy

\section{OpenEdition}

Journals

Édition électronique

URL : http://journals.openedition.org/formationemploi/1621

DOI : $10.4000 /$ formationemploi.1621

ISSN : 2107-0946

Éditeur

La Documentation française

Édition imprimée

Date de publication : 1 avril 2007

Pagination : 123-139

ISSN : 0759-6340

Référence électronique

Jean-Étienne Bidou et Isabelle Droy, «Quel espace de choix des métiers ? Les inégalités d'accès aux savoirs techniques en Guinée Maritime », Formation emploi [En ligne], 98 | avril-juin 2007, mis en ligne le 30 juin 2009, consulté le 30 octobre 2020. URL : http://journals.openedition.org/formationemploi/ 1621 ; DOI : https://doi.org/10.4000/formationemploi.1621 


\title{
Numéro spécial
}

\section{Quel espace de choix des métiers? Les inégalités d'accès aux savoirs techniques en Guinée Maritime}

par Jean-Étienne Bidou et Isabelle Droy*

\author{
Une analyse de l'accès aux formations met en évidence les difficultés \\ de transformation des libertés formelles en libertés réelles \\ dans l'accès aux savoirs, ce qui conditionne ensuite l'accès à des métiers \\ plus ou moins valorisants et rémunérateurs.
}

Le système de production de Guinée Maritime (Guinée-Conakry) a été décrit comme celui d'une multiactivité opportuniste où la complémentarité des activités est recherchée par les ménages, car elle permet l'adaptation et l'ajustement permanent à un contexte écologique, social et économique mouvant (Rossi et alii, 2002). Mais si chaque ménage cherche à diversifier ses sources de revenu, cela ne signifie pas forcément que chacun ait une égale liberté d'accès aux savoirs, aux apprentissages et aux ressources qui vont permettre d'exercer ces diverses activités suivant ses compétences et ses choix personnels.

En effet, le milieu rural de Guinée Maritime présente des traits spécifiques qui conduisent à s'interroger sur la liberté des individus «à faire et à être » (Sen, 1999). Tout d'abord, l'acquisition de savoirs techniques à travers un cadre formel d'enseignement est très minoritaire, faute de structures adéquates mises en place par les politiques de formation professionnelle. Ensuite, le milieu est marqué par une grande pauvreté qui entrave le développement des différentes activités. Enfin, les inégalités de sexe et de statut pèsent fortement sur les possibilités de choix des individus.

* Jean Étienne Bidou est maître de conférences en géographie et rattaché à L'UMR 5185 ADES. II travaille sur les systèmes ruraux en Afrique au sud du Sahara et s'intéresse aux questions de risque et de vulnérabilité. Par ailleurs, il est codirecteur de la revue « Éducation relative à l'environnement ". I| a publié notamment : Bidou J.E. (2003), «Éducation relative à l'environnement ou acculturation? », Éducation relative à l'Environnement, n 4, Montréal, pp. 8-25. Bidou J.E. et Gbéré Touré J. (2002), " La population de la Guinée - dynamiques spatiales », Cahiers d'Outre-Mer, pp. 9-30. 
Isabelle Droy est chargée de recherche à l'IRD, UMR C3ED (Institut de recherche pour le développement-université de Versailles St Quentin en Yvelines). Elle a travaillé sur la conception et la mise en place d'observatoires socio-économiques en milieu rural (Madagascar, Guinée). Elle effectue, à partir de ces observatoires, des analyses sur la pauvreté, la vulnérabilité et les inégalités, plus particulièrement les inégalités de genre. Elle a notamment publié : Droy I. (2005), "Pauvreté, enclavement et scolarisation en milieu rural guinéen », Mondes en développement, vol. 33-2005/4, n० 132 ; «Éducation et développement», pp. $111-127$. Droy I. (2006), "Quel apport de l'approche par les capabilités pour l'analyse des inégalités de genre? Amartya Sen, un économiste du développement», V. Reboud Éd. AFD, Notes et documents, $n^{\circ} 30$.

Dans ces conditions, il est intéressant de mobiliser l'approche par les capacités ${ }^{1}$ développée à partir des travaux d'A. Sen pour analyser les droits et libertés réels des individus à se former et à accéder aux activités qu'ils souhaitent exercer.

Après avoir rappelé le contexte de l'étude et l'apport de la théorie des capacités dans la problématique de l'accès aux savoirs, nous présentons les traits saillants de l'organisation sociale en milieu rural guinéen. Avec la présentation de la méthodologie de l'étude, nous explorons le lien entre les conditions de choix des activités et les statuts sociaux, marqués par de fortes inégalités dans cette société. Nous comparons ensuite les différentes activités sous l'angle de leur valorisation monétaire. Enfin, nous abordons la question de l'accès à l'école comme moyen d'élargir l'espace de choix des activités.

\section{APPROCHE PAR LES CAPACITÉS ET ACCÈS AUX SAVOIRS}

Les méthodes d'analyse de la pauvreté se sont diversifiées depuis quelques années, répondant en cela à une tentative de mieux appréhender les différentes facettes d'un phénomène complexe. Les approches quantitatives

\footnotetext{
${ }^{1}$ Le mot anglais « capability» (A. Sen) est difficilement traduisible en français. Il décrit l'aptitude à utiliser ses capacités pour faire quelque chose ou être dans un état particulier. Il sera donc ici traduit soit par le néologisme «capabilité » ou, par souci de simplicité, par " capacité » dans les expressions "approche ou analyse par les capacités » ou « développement des capacités ».
}

ne sont plus limitées à la seule analyse monétaire et l'approche par les capacités, développée à partir des travaux d'Amartya Sen, génère la possibilité de nouveaux approfondissements méthodologiques en prenant en compte des dimensions jusque-là occultées. Les rapports sur le développement humain du PNUD (programme des nations unies pour le développement) et certaines politiques de lutte contre la pauvreté y font aussi maintenant explicitement référence.

\section{L'approche par les « capacités »}

Dans l'approche utilitariste du bien-être, les actions des individus ont pour objectif d'améliorer leur propre bonheur et leur intérêt personnel (regroupés sous le vocable « utilité »). La mesure du bien-être est réalisée a posteriori par le biais du niveau des dépenses en biens de consommation (les préférences observées). Les approches monétaires de la pauvreté se réfèrent à ce cadre théorique. Les ménages sont ensuite classés en fonction de leur revenu, mesuré directement ou à travers les dépenses effectuées. Le classement des ménages par rapport à un revenuseuil définit les populations pauvres et non pauvres.

Le modèle utilitariste a été remis en cause par de nombreux auteurs, comme John Rawls (1971) dans son ouvrage "Théorie de la justice». Puis, les travaux de Sen ont poursuivi et élargi cette critique en permettant de mieux comprendre les aspects multidimensionnels du bien-être. L'idée fondamentale de l'approche par les capacités est de comprendre ce que l'individu parvient réellement à faire et être avec les biens et les caractéristiques dont il dispose, ce que Sen désigne par « fonctionnements ».

En effet, Sen montre que les informations dont on dispose sur les caractéristiques d'un bien ne permettent pas nécessairement de savoir ce que l'individu va pouvoir accomplir avec ce bien (Sen, 1987, 1992, 1993 ; Silber, 2001). La manière d'utiliser ce bien dépendra des caractéristiques personnelles de l'individu, de ses acquis, et des droits qu'il a dans la société où il vit². La relation entre les droits ou les

\footnotetext{
2 L'exemple de la bicyclette est le plus souvent cité, car il est très explicite. Posséder une bicyclette ne permet pas de déduire que la personne peut réaliser le fonctionnement «pouvoir se déplacer plus rapidement qu'à pied » : d'une part, il faut savoir l'utiliser et d'autre part en avoir le droit, ce qui n'est pas possible pour les femmes dans de nombreuses sociétés.
} 
ressources dont disposent les individus et l'usage qu'ils en font, correspond pour Sen à la conversion des libertés formelles en libertés réelles. La prise en compte des droits et obligations auxquels sont soumis les individus au sein de leur société est donc essentielle pour analyser leurs choix.

L'approche développée par Sen dans les années 80 a été poursuivie par d'autres chercheurs, comme la philosophe Martha Nussbaum³.

\section{Potentialité et accessibilité freinent la constitution des fonctionnements}

Dans chacune des dimensions du développement humain, un individu ou un groupe va être confronté à deux problèmes : l'inégalité des potentialités et celle de l'accessibilité à ces potentialités.

En premier lieu, chaque individu possède un «stock d'actifs » particulier, lié à ses caractéristiques personnelles, à son éducation, à son état de santé, à son capital physique et financier, aux relations sociales qu'il entretient, etc. On appelle ces actifs les potentialités. La pauvreté des potentialités exprime donc un déficit d'accumulation dans les domaines de la santé, de l'éducation, des biens matériels ou des relations sociales. (Dubois, Mahieu, Poussard, 2001).

Les différentes potentialités sont regroupées sous le terme de « capital », qui illustre bien l'aspect d'accumulation à la fois pour les personnes et pour la sociétét. Le capital physique et financier est bien entendu la forme la plus connue : il fait référence aux biens matériels (terres, équipements agricoles, matériel etc.) et aux actifs financiers que possède un individu. On y inclut aussi la possibilité d'accéder au crédit. Le capital humain est bien analysé depuis les années 90 , date des premiers rapports sur le développement humain élaborés sous l'égide du

\footnotetext{
${ }^{3}$ A. Sen parle de "capability» au sens générique alors que M.Nussbaum se réfère à une liste fermée de «capabilities ». On ne détaillera pas ici les différences théoriques entre Sen et Nussbaum, les auteurs s'inscrivant dans l'approche de Sen qui décrit l'espace des capabilités comme celui de «la liberté à faire et à être ». Comme telles, elles sont enchâssées dans la culture locale, qui, loin de garantir les capacités centrales de Nussbaum, n'assure même pas l'égalité des statuts des personnes.

${ }^{4}$ En termes économiques, le capital est un bien résultant d'une accumulation, qui est utilisé pour produire un revenu et qui s'use à travers cette utilisation. Le capital humain permet d'obtenir un salaire sur le marché du travail, le capital social produit un revenu social comme les transferts. La vision sociologique met plus l'accent sur les avantages sociaux tirés de cette potentialité : intérêt de l'éducation pour guider ses choix, utilité des réseaux sociaux, etc.
}

PNUD. De nombreux programmes de développement tentent de le renforcer, à travers l'éducation et la santé. Les analyses montrant l'importance du capital social sont plus récentes, elles ont été développées à la suite des travaux de Pierre Bourdieu qui a révélé l'importance des réseaux de relations sociales dans la réussite économique des individus. On peut parler, par analogie, de capital politique en faisant référence à la pratique de la démocratie. De même, le capital d'éthique rassemble l'ensemble des normes et valeurs qui visent au respect des droits des personnes et des communautés.

En second lieu, l'accès à un certain nombre de biens, de services, d'informations ou la participation aux décisions ne sont pas les mêmes pour tous. Certains groupes peuvent être privés de droits ou de possibilités d'accès à la propriété foncière, au crédit ou à certains services comme l'éducation ou la santé, soit par le fait d'une discrimination sociale (selon le sexe, selon l'ethnie) ou du fait des inégalités spatiales et économiques (absence d'école ou de dispensaire à proximité). On parle alors de pauvreté d'accessibilité.

Ainsi, les potentialités ou la mesure du stock des actifs ne sont pas suffisantes pour mesurer le bienêtre, car encore faut-il que l'individu ait la possibilité de les activer ou de les convertir en fonctionnement. La conversion est conditionnée par les caractéristiques personnelles des individus, mais aussi, le plus souvent, par les contraintes sociales. Ces contraintes d'accès revêtent de multiples formes comme, par exemple, l'impossibilité d'écouler sa production faute $d$ 'accès routier, d'être soigné faute de personnel soignant, mais aussi d'exercer telle activité particulière en raison de son sexe ou de son groupe social. Chaque fonctionnement va ainsi dépendre de ces deux paramètres, potentialité et accessibilité.

La capabilité d'une personne se définit alors par «les différentes combinaisons de fonctionnements qu'il lui est possible de mettre en cuvre. Il s'agit d'une forme de liberté [...], la liberté de mener des modes de vie divers» (Sen, 2000). L'ensemble des capabilités correspond donc alors aux fonctionnements accomplis et non accomplis, mais que l'individu pourrait choisir s'il le voulait ou s'il le devait, à la suite d'une perturbation altérant ses autres fonctionnements. Par exemple, en cas de destruction des récoltes, un agriculteur homme ayant été scolarisé 
aura plus de facilités pour trouver une activité rémunératrice alternative, en migrant, qu'une femme non alphabétisée. Cette dernière voit sa capabalité réduite par le fait de n'avoir pas eu accès aux savoirs formels dispensés par l'École et, de surcroît, sa mobilité est plus contrainte que celle des hommes (Droy, 2006). Les notions de libertés et de droits sont donc au cœur de l'analyse par les capacités.

\section{L'inégalité d'accès aux savoirs et à la formation}

Dans un contexte de libéralisation et de monétarisation de l'économie, comme celui que connaît la Guinée actuellement, les fonctionnements « maîtriser des savoirs formels $»^{5}$ et « être en bonne santé », ont une valeur intrinsèque. La liberté d'accès à l'éducation apparaît chez A. Sen « comme une liberté instrumentale, permettant d'accroître les capacités et les autres libertés, indispensables au développement humain » (Poirot, 2005). L'individu devient ainsi plus autonome et accroît le champ de ses libertés. Dans cette perspective, qui s'inscrit dans la pensée de Sen, l'accumulation de capital humain-formation doit être considérée dans un sens plus large que la seule amélioration des capacités productives des individus ${ }^{6}$.

L'approche des capacités distingue trois groupes de facteurs de conversion, les facteurs individuels, environnementaux et sociaux, (Robeyns, 2005) qui s'avèrent particulièrement pertinents pour analyser la réalisation $\mathrm{du}$ fonctionnement «être instruit et formé ». La constitution du capital de formation peut se faire dans le secteur formel, comme à l'école ou dans un centre de formation professionnelle, ou bien dans le secteur informel dans le cadre d'un apprentissage familial ou chez un artisan.

A) Les facteurs de conversion personnels constituent les caractéristiques personnelles qui varient d'un individu à l'autre : l'aptitude et l'envie d'apprendre, l'état physique de l'individu (une personne handicapée physique aura plus de difficultés pour certains apprentissages).

\footnotetext{
${ }^{5}$ Comme la lecture ou l'écriture : même en zone rurale très enclavée, la maîtrise de ces outils facilite les liens devenus nombreux avec le monde extérieur.

${ }^{6}$ Pour certains économistes, le capital humain est souvent analysé uniquement sous cet angle assez étroit.
}

B) Les facteurs environnementaux de conversion sont conçus au sens large et englobent les caractéristiques géographiques et l'équipement du territoire concerné. Dans un pays comme la Guinée, il existe un déficit d'offre structurée de formation, qui se manifeste par l'absence d'écoles ou de centres de formation professionnelle à proximité du lieu de résidence du ménage. Dans le cas où l'offre existe (écoles primaires), encore faut-il que les ménages aient la possibilité financière de faire face aux coûts de scolarisation (frais de scolarité, mais aussi d'hébergement) et qu'ils puissent se priver de la force de travail que représente une personne du ménage, fût-elle encore enfant. Cela dépend donc du capital physique ou financier dont dispose le ménage.

C) Les facteurs sociaux de conversion sont liés aux politiques publiques, aux normes sociales, aux pratiques discriminantes, aux relations de pouvoir. Ils forment une partie importante de la liberté réelle des individus, souvent beaucoup plus restreinte que la liberté formelle qui renvoie aux principes d'égalité d'accès à l'éducation et à la formation quels que soient le sexe et le groupe social (Guérin, 2003). Dans les sociétés fortement hiérarchisées, la liberté réelle de choix et d'accès aux savoirs est réduite pour certaines catégories de population. Cet accès différentiel aux formations, et ensuite aux activités qui y sont liées, véhicule la reproduction des inégalités, qu'elles soient sociales ou économiques : certains métiers sont reconnus socialement, mais ne procurent pas une rémunération attractive; pour d'autres métiers, c'est l'inverse, mais plus fréquemment, reconnaissance sociale et économique sont étroitement liées.

L'espace des capabilités est cependant difficile à appréhender, puisqu'il englobe les fonctionnements non accomplis, mais qui pourraient l'être. La mesure de la « liberté à faire et à être » est tout aussi délicate. On ne peut donc avoir qu'une vision partielle de cet espace, à travers les fonctionnements accomplis, mais aussi à travers certains actifs qui sont mesurables. Malgré ces difficultés, cette approche est particulièrement pertinente par rapport à notre questionnement sur l'accès aux savoirs et aux métiers en Guinée. Elle permet en effet d'établir une grille de lecture qui prend en compte les contraintes sociales qui conditionnent les capacités de conversion, ainsi que l'espace de liberté de choix des activités. 


\section{STRATÉGIES DE DIVERSIFICATION ET INÉGALITÉS SOCIALES}

\section{Multiactivité et réduction des risques}

Le milieu rural de Guinée Maritime ${ }^{7}$ est caractérisé par la diversité des activités : en plus de l'activité principale orientée vers l'agriculture, les actifs ont souvent une deuxième, voire une troisième activité, pratiquée en même temps ou en alternance avec l'activité agricole. Celle-ci est centrée sur la riziculture, qu'elle soit pluviale ou inondée. Ni l'un ni l'autre de ces modes de culture n'épargne aux ménages une longue période de soudure 8 qui rend nécessaire la recherche d'autres sources de revenu.

La multiactivité assure ainsi la stabilisation du système de production. Non seulement elle amortit les effets de la soudure alimentaire, mais elle procure de l'argent dans une économie de plus en plus monétarisée, alors que l'agriculture ne génère que peu de surplus commercialisables.

Ainsi, la pêche, la saliculture et de nombreuses activités artisanales constituent autant de composantes de ce système, qui permet aux ménages de limiter et de contrôler les risques. La saliculture est une activité ancienne, pratiquée traditionnellement par les femmes. La pêche est pratiquée par les hommes ou par les femmes, dans des milieux divers et suivant des techniques variées. Autrefois, il s'agissait uniquement d'une activité de saison creuse. Si elle le reste pour une grande partie de la population, on voit cependant l'émergence d'un groupe de pêcheurs professionnels.

Reste une multitude d'autres activités qui sont exercées individuellement par les différents membres du ménage, d'une façon régulière, mais aussi sur des périodes courtes, quelquefois de façon épisodique lorsque le besoin s'en fait sentir (comme la cueillette). Elles font partie des savoir-faire du ménage et constituent des ressources utilisables si nécessaires, ce qui est une caractéristique des fonctionnements décrits

\footnotetext{
${ }^{7}$ La Guinée maritime est l'une des quatre régions naturelles de Guinée. C'est une plaine côtière de $300 \mathrm{~km}$ de long et de 50 à $90 \mathrm{~km}$ de large, bordée par l'Océan Atlantique à l'ouest et le pied de la falaise du Fouta-Djalon à l'est.

8 La soudure est la période, avant une récolte, où les vivres viennent à manquer si la récolte précédente n'a pas été suffisante. Une longue soudure peut dégénérer en disette, voire en famine.
}

par Sen: en cas de choc, comme une mauvaise récolte, les ménages sont capables de réactiver une activité qu'ils ne pratiquent qu'épisodiquement.

Dans ces conditions, l'accès aux apprentissages techniques est un aspect crucial de la reproduction du système.

\section{Une société fortement inégalitaire}

Les populations de Guinée Maritime forment des groupes divers, et d'importance démographique inégale. On préfère souvent parler à leur propos de groupes sociaux que d'ethnie, notion toujours trop figée pour décrire ces populations dont les caractères culturels ont intégré des apports de nombreux groupes migrants9 (Bouju, 1999). Chacun de ces groupes a évolué en lien avec les autres, mais selon sa dynamique propre; chacun a sa langue, et quelquefois même plusieurs. Cette histoire leur confère à la fois des différences affirmées, mais aussi des formes sociales communes. Ainsi, on distingue trois formes d'inégalités, présentes dans tous les groupes, mais dont l'intensité est plus ou moins marquée : inégalités internes à la famille, inégalités de sexe et entre les divers groupes sociaux à l'intérieur du village.

\section{Les cadets sociaux ou les inégalités de statut intrafamilial}

L'organisation familiale est de type patrilinéaire, en faveur de l'aîné masculin, en termes de règles de descendance, de statut et d'héritage. Ces familles étendues sont des fragments de lignage et rassemblent trois ou quatre générations autour d'une même concession ${ }^{10}$, qui est souvent marquée spatialement par un lieu commun d'habitat. L'aîné, qui est le chef de concession, étend son autorité sur ses enfants, y compris quand ceux-ci sont mariés, mais aussi sur ses neveux et dans une certaine mesure, sur ses frères moins âgés. Il

\footnotetext{
${ }^{9}$ Les historiens distinguent trois vagues de peuplement. Une migration ancienne, probablement entamée dès le $\mathrm{XV}^{\mathrm{e}}$ siècle et qui s'est prolongée beaucoup plus tard. Elle concerne surtout les baga, eux-mêmes divisés entre les landouma et les nalou. Une deuxième vague concerne essentiellement les soussou, descendus du Fouta Djallon à partir du XVI ${ }^{\mathrm{e}}$ siècle, de langue et de culture mandé. Ils forment le groupe le plus important et tendent à assimiler les autres groupes sur leurs marges. Le XIXe siècle voit arriver d'autres groupes : des peuls, peu nombreux, mais dont le royaume a contrôlé une partie de la côte, et surtout les diakhanké, de langue mandé, venus à la côte comme guerriers et prêcheurs musulmans. ${ }^{10}$ La concession est une unité de résidence où résident plusieurs ménages, ayant chacun leur propre partie du logement dans la concession.
} 
dispose de droits d'accès et de contrôle sur les ressources foncières et organise le travail sur les champs collectifs ; il gère ses biens propres mais aussi, en partie, ceux de ses dépendants. Les membres de la concession qui sont sous son autorité sont des « cadets sociaux ", aussi bien à l'intérieur du groupe qu'à l'extérieur et l'aîné est parfois le seul à avoir le droit de participer à la vie publique du village. Ainsi, sans être généralement un despote familial, le patriarche dispose d'un pouvoir de coercition considérable sur les membres de sa concession (Meillassoux, 1975).

\section{Les différences de sexe, transversales et omniprésentes}

Les différences entre garçons et filles, hommes et femmes, sont omniprésentes et se superposent aux inégalités de statut au sein de la famille. La polygamie est la norme, quoique le cas le plus fréquent soit la bigamie. Pour avoir une troisième, voire une quatrième épouse (fait rare dans la région), il faut être riche. Les femmes, toujours «mineures» par rapport aux hommes, ne sont pas pour autant égales entre elles : la première épouse du chef de concession est nettement mieux considérée que la seconde épouse du cadet des neveux du chef. Les femmes ont une autonomie financière réduite et leurs biens sont gérés par leur époux ou leur frère ; en cas de veuvage, la pratique du lévirat, qui consiste à épouser le frère de l'époux défunt, prévaut.

La polygamie engendre et maintient une répartition particulière des droits et obligations au sein du ménage et vis-à-vis des enfants : il n'y pas de mise en commun des ressources, et les responsabilités de chacun sont rigoureusement déterminées. En plus de devoir assurer quotidiennement «la sauce» qui accompagne le riz fourni en principe par l'homme, les femmes doivent le plus souvent assumer la plupart des charges liées aux enfants; or, ces dernières sont de plus en plus monétarisées. Il faut de l'argent pour acheter des vêtements, assurer les frais de scolarité et ceux liés à la santé. Les femmes exercent ainsi une série d'activités rémunératrices, pour lesquelles elles essaient de garder une partie du revenu.

\section{Métiers castés et métiers libres}

Dans le contexte de bouleversements profonds qu'a connu la Guinée depuis 1984, qui correspond à la mort de Sékou Touré et à la politique d'ouverture et de libéralisation économique, il est nécessaire de s'interroger sur les transformations des métiers. En effet, si on observe dans les villages des activités dont les techniques ne semblent pas avoir évolué depuis des décennies, on voit aussi apparaître de nouvelles activités, liées à l'introduction des techniques ou outils venus de l'extérieur (moto, vélos, radio, etc.).

\section{- Les activités « traditionnelles»}

Ces activités sont celles dont les techniques ont peu évolué, comme la vannerie, la poterie ou la forge. On trouve dans ce groupe une part importante de métiers liés à des groupes castés (Kyburz, 1997).

En effet, la Guinée Maritime, comme la plupart des régions assimilées ou influencées par la culture mandingue, est marquée par une forte hiérarchisation entre hommes libres, métiers castés, et, autrefois, captifs. Les métiers castés transforment des matériaux provenant de la nature, comme le minerai de fer, le bois, la fibre textile ou l'argile. Et dans la religion animiste, toujours présente malgré l'islamisation, ces matières sont porteuses d'un souffle vital, le nyama (Person, 1968). Leur manipulation et leur transformation exigent non seulement des savoirs manuels, mais aussi la maîtrise des codes pour le dialogue avec la surnature. Ces savoirs sont transmis au sein de la caste, qui est régie par une endogamie stricte. La plus nombreuse et la plus connue est celle des forgerons fabriquant tous les objets en fer, les bijoux, mais aussi quelquefois les objets en bois et les instruments de musique; leurs femmes sont des potières. Ils ont aussi des fonctions religieuses, et la caste s'occupe des rites d'initiation que sont la circoncision et l'excision. L'importance des relations avec la surnature, l'enchâssement de leurs activités dans la culture traditionnelle induisent des contraintes sociétales fortes. Ces groupes sont à la fois craints et méprisés par le reste de la population. On imagine bien dans ce cas que la « liberté à faire et à être ", à mener une vie digne, qui sont les fondements de l'approche des capacités, est restreinte.

\section{- Les activités « intermédiaires »}

Avec la modernisation, certains métiers se sont transformés : ils répondent à des besoins qui sont anciens, comme la construction des habitations, mais en 
utilisant des matériaux modernes comme la tôle ou le ciment pour les maçons. Ceux qui exercent ces métiers disposent de plus de liberté que pour les métiers castés, aussi bien dans les conditions d'entrée et d'exercice du métier que dans leurs relations avec le reste du village.

\section{- Les activités « modernes »}

Enfin, on trouve des métiers modernes qui répondent à des besoins nouveaux et mettent en œuvre des matériaux souvent importés, comme les mécaniciens ou les réparateurs de vélo. Cependant, ces besoins modernes sont encore assez peu intenses dans les campagnes, en raison notamment du très faible niveau de vie en milieu rural. L'indicateur de développement humain du PNUD de 2006 classe la Guinée à la $160^{\mathrm{e}}$ place sur 177 pays. La Guinée fait partie des pays pauvres très endettés.

Cette classification en trois grands groupes nous a servi de fil conducteur pour l'analyse de l'enquête réalisée en Guinée Maritime en 2004.

\section{L'IMPORTANCE DE LA FAMILLE DANS L'ACCÈS AUX APPRENTISSAGES}

\section{Méthodologie et présentation de l'enquête}

Les résultats présentés sont issus d'une recherche menée dans le cadre de l'Observatoire de la Guinée Maritime ${ }^{11}$, qui est un projet pluridisciplinaire de recherche-action, axé sur le milieu rural, ayant pour objectif de produire des outils méthodologiques permettant l'amélioration de la maîtrise locale de la gestion des ressources naturelles. Le volet «pauvreté et systèmes d'activités », dont les données sont mobilisées ici, a pour objectif de fournir une meilleure connaissance de l'organisation des activités, des conditions de vie et des revenus des ménages.

\footnotetext{
${ }^{11}$ L'Observatoire de la Guinée Maritime (OGM) est un projet conduit en partenariat par l'université Michel de Montaigne-Bordeaux III et l'AFVP (Association française des volontaires du progrès) pour le compte du ministère guinéen du Plan et financé par le FFEM (Fonds français pour l'environnement mondial) et l'AFD (Agence française de développement). Il est dirigé par le professeur Georges Rossi de l'université de Bordeaux III. L'équipe du « volet pauvreté » est composée d'I. Droy, J.-L. Dubois, D. Leyle et A. Soumah. L'enquête « savoirs et apprentissages » a été conçue et réalisée par J.-E. Bidou, I. Droy et A. Soumah.
}

L'encadré 1 présente l'organisation de l'enquête et l'échantillon.

Le principe est le suivant: le point de départ est une enquête réalisée auprès de 845 ménages, intitulée la base « pauvreté ». À partir de cette enquête, un sous-groupe a été créé pour constituer la base «multiactivité »: les ménages sélectionnés pour cette base sont ceux dont au moins une personne pratique une activité qui n'est pas agricole ou d'élevage ; on a exclu aussi de cette sélection les quelques salariés du secteur public (instituteur, infirmier...). Une approche comparative est donc possible entre les sous-groupes. Les individus retenus dans la sélection de la base "multiactivité » pratiquent des activités artisanales que l'on a classées comme « traditionnelles » (forgeron, potier, tisserand ou encore artisanat alimentaire, « intermédiaires » comme maçon, ou « modernes » comme réparateur de vélo, ou mécanicien etc. L'activité de pêche en mer (différente de celle de la pêche continentale) appartient aux activités modernes (utilisation de pirogue à moteur, de filets en polyamide), mais elle a été traitée à part en raison de son importance économique dans la région.

Ces activités sont pratiquées en activité principale ou en activité secondaire, et ont éventuellement fait l'objet d'une formation complémentaire. La majorité $(59 \%)$ des ménages de la base "pauvreté » a au moins une personne qui est concernée par cette sélection (941 personnes au total). Les individus de l'échantillon sont essentiellement des adultes de plus de 18 ans (89\%). Les hommes représentent les deux tiers de ce groupe (62\%) alors que dans l'ensemble de la population adulte, ils ne sont que $43 \%$.

Le tirage d'un échantillon de la base «multiactivité » a permis de mener une enquête qualitative complémentaire « récits de vie » : l'objectif était de reconstituer par des entretiens les itinéraires de formation et d'accès aux métiers de 53 individus. Cette enquête a permis d'approfondir les conditions de l'apprentissage des métiers sélectionnés dans cette étude.

\section{Métiers choisis, métiers imposés}

La notion de choix et de liberté est une composante essentielle de l'approche des capacités et pourtant elle est difficilement mesurable par les enquêtes classiques sur la pauvreté, qui reposent sur des questionnaires à choix fermé. Au mieux, ces enquêtes ne 
peuvent mesurer que les fonctionnements accomplis (formation suivie, nombre d'années d'étude, biens matériels possédés, etc.). Pour connaître la liberté de choix du métier et les itinéraires d'accès aux apprentis- sages, il est nécessaire de s'entretenir individuellement avec les personnes concernées. À partir de cette enquête « récits de vie », les itinéraires de formation ont été reconstitués de la façon suivante :

\title{
Encadré 1 \\ Données sur les savoirs et apprentissages : organisation de l'enquête et échantillonnage
}

\author{
Zone d'enquête en milieu rural de Guinée Maritime sur $\mathbf{4}$ communes rurales de développement \\ (CRD) \\ - Kanfarandé pour la région la plus isolée, proche de la Guinée-Bissau ; \\ - Mankountan au sud de Kanfarandé et d'accès assez aisé depuis Conakry ; \\ - Tougnifily avec un important marché ; \\ - Boffa : une partie reliée à la capitale par une bonne route, une partie insulaire.
}

Zone de travail de l'Observatoire de la Guinée Maritime.

Sélection de 9 sites d'enquêtes (villages) sur la base d'un choix raisonné :

degré d'enclavement, zone maritime ou continentale, groupe culturel dominant, système d'activités

\section{Base pauvreté}

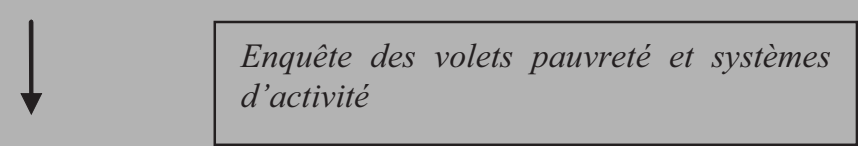

Enquête exhaustive auprès des ménages des 9 sites :

845 ménages enquêtés avec un questionnaire axé sur les activités, les revenus, les conditions de vie. Sur certains modules comme celui des activités, les renseignements sont recueillis à l'échelle individuelle au sein du ménage. Cette enquête forme la base de données " pauvreté ».

\section{Base multiactivité}

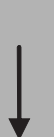

Recherche sur la multiactivité et les accès aux apprentissages.

Sélection dans la base pauvreté des ménages dont au moins un individu pratique une activité qui n'est ni agriculteur et/ou éleveur, ni une activité salariée du secteur public (instituteur, infirmier...).

941 individus enquêtés dans la base «pauvreté » pratiquent ces activités; cela concerne $59 \%$ des ménages de la base pauvreté. Cette sélection forme la base « multiactivité ».

\section{Récits de vie}

À partir de la base multiactivité, tirage d'un échantillon de $\mathbf{5 3}$ individus (dont $39 \%$ femmes) pour des entretiens ouverts : récits de vie de leur expérience d'accès au métier. Choix raisonné incluant le genre, le type de métier, l'accès à une formation complémentaire. Cette sélection est appelée " récit de vie ». 


\section{Le poids de la collectivité}

Pour un peu moins de la moitié des enquêtés, le choix de l'activité revient à la famille, par ce qu'ils appellent la «tradition familiale» ou la «décision familiale ».

"Je suis née dans une famille de forgerons où la poterie est une tradition pour les femmes. Ma mère est griote, moi j'ai appris ce métier à côté de ma bellemère issue d'une famille de forgerons; ce métier est un moyen de subsistance pour les femmes de forgerons. Ma belle-mère m'apprenait graduellement ce travail dont on acquiert la connaissance en fonction de l'âge car cela fait partie de notre éducation de base, en plus des travaux ménagers. Les garçons à la forge et les filles apprennent la poterie à côté de nos mères. » Femme, Potière, Kankayani, 35 ans

Cet entretien illustre bien combien les choix d'activité et de conjoint sont restreints pour les groupes castés. Ce qu'on appelle « tradition familiale » correspond ici en réalité à l'appartenance à une catégorie sociale. Dans ces situations, les facteurs sociaux de conversion sont donc extrêmement contraignants.

Ils peuvent l'être moins pour d'autres groupes sociaux, où le choix du métier n'est pas imposé, mais fortement guidé par la famille. En effet, celle-ci maîtrise un réseau social permettant l'acquisition des savoirs et quelquefois l'exercice de l'activité. Les métiers sont des activités «traditionnelles»: pêcheur, vannier, guérisseur, fabricant de pirogue pour les garçons, l'artisanat alimentaire, le petit commerce et le fumage $\mathrm{du}$ poisson pour les filles. S'y ajoutent quelques métiers «intermédiaires» lorsque les parents l'exerçaient ; maçons, menuisiers ou tailleurs, euxmêmes liés au bois, à la pierre ou à la fibre textile.

Pour les uns comme pour les autres, l'âge de début de l'apprentissage est très précoce: environ quatre sur dix ont commencé à apprendre le métier avant dix ans et ils sont huit sur dix à avoir débuté l'apprentissage avant 16 ans. La précocité de cet apprentissage limite les options de l'enfant et compromet l'accès à l'école.

Ce type de choix pose bien sûr la question du poids des structures familiales, lignagères et même villageoise et, en regard, l'autonomie du sujet (Marie A., 1997). Dans ce contexte, les préférences ou les carac- téristiques personnelles entrent peu en ligne de compte; seul le statut de l'enfant à l'intérieur du groupe détermine ses marges de manœuvre.

\section{La décision individuelle}

Pour plus de la moitié, la motivation individuelle est plus importante: le métier a été choisi parce qu'il plaît à la personne ou qu'il permet de "gagner de l'argent». Ce sont des métiers modernes: chauffeurs, mécaniciens et réparateurs divers ainsi que les métiers du bâtiment où l'on a dû chercher un apprentissage en dehors du village.

Dans l'exemple suivant, comme dans beaucoup d'autres, les personnes réalisent leur projet en fuyant le milieu familial, ou en s'y opposant :

"J'ai décidé d'apprendre un métier pour ne pas souffrir. Je suis sorti de chez moi sans prévenir mon père. C'est après trois ans d'apprentissage qu'il a été informé de ma destination. J'ai appris la couture pendant 5 ans à Kolaboui.» Homme, Tailleur, Madya, 51 ans

Ici, la personne revendique son autonomie face au milieu social et familial et fonde sa « liberté à faire et à être ». Cette décision n'est cependant envisageable que pour les hommes et marque la nette différence de sexe en matière de facteurs de conversion.

Dès lors, le choix du métier dépend du goût et surtout des opportunités. L'âge de début de l'apprentissage est plus tardif que pour le premier groupe, et seulement 3 sur 10 ont commencé l'apprentissage avant l'âge de 15 ans.

Cependant, la pression de la famille peut s'exercer aussi une fois le métier appris, même chez les adultes :

«Pour me faciliter les travaux, j'ai appris la traction animale auprès d'un voisin du village qui a été formé par une $O N G$ (organisation non gouvernementale) $a ̀$ Amsinia. C'est à la suite de cette formation que j'ai pris trois boufs en location plus la charrue pour faire mon champ. Je faisais trois jours chez le patron et un jour chez moi. Mais mon frère, qui est à Conakry, n'était pas d'accord avec cette méthode de travail, il la jugeait comme l' "exploitation de l'homme par l'homme". Mon frère me disait que je servais de main-d'œuvre, donc sous sa pression qui 
grandissait tous les jours, j'ai abandonné cette activité. » Homme, Maçon, Brika, 44 ans

La rhétorique marxiste utilisée par le frère aîné, tout droit issue de l'époque de Sékou Touré, masque un problème plus sérieux, sur lequel se sont heurtées les politiques de développement. En effet, en Guinée Maritime, les labours, toujours collectifs et manuels, constituent à la fois une mobilisation de la force de travail et la célébration du groupe. La traction attelée, bien que représentant un progrès agricole, est perçue comme une menace pour la collectivité parce qu'elle permet un accroissement de la productivité du travail individuel. C'est donc un homme dangereux qui a été remis sur le droit chemin par son aîné.

\section{La famille, principal lieu} d'apprentissage technique

Les structures formelles de formation professionnelle sont quasiment inexistantes dans la région d'enquête, comme d'une manière générale en Guinée. La transmission des savoirs techniques et la construction des capabilités s'opèrent donc essentiellement dans un environnement non formel, dans le cadre d'un apprentissage au sein de la famille ou d'un placement chez un artisan. Pour plus de huit personnes sur dix de l'échantillon enquêté, c'est essentiellement dans le cadre familial que se transmettent les savoirs techniques. Pour les deux tiers, cet apprentissage familial a lieu le plus souvent dans le ménage ${ }^{12}$, mais aussi parfois en ville chez un membre de la famille.

«Dans le souci d'apprendre un métier pour ma vie, pour ne pas me contenter seulement des travaux champêtres, je suis allé à côté de mon frère à Kamsar pour m'aider dans ce sens. Il n'y avait pas beaucoup de choix, lui-même était chauffeur, il m'a mis dans ce métier. Je suis resté dans son camion pendant un an. " Homme, Chauffeur, Kambilam, 49 ans

Seuls huit enquêtés sur cinquante trois ont été formés chez un artisan comme apprenti ou, plus rarement, par une association. Ces deux types de formation sont plutôt réservés aux hommes, les femmes restant dans le cadre du ménage pour apprendre une nouvelle

12 Souvent les femmes distinguent l'apprentissage dans leur ménage d'origine et l'apprentissage dans le «ménage conjugal » après le mariage. Compte tenu de la taille réduite de l'échantillon, nous avons tout rassemblé ici. activité. Une fois encore, les inégalités de sexe limitent les facteurs de conversion pour les femmes.

Le coût de ces formations est compensé généralement par une contrepartie en travail, plus rarement en nature ou en argent. Les raisons du choix de l'activité sont plus individuelles ou économiques.

Quand l'apprentissage nécessite un hébergement en dehors du ménage d'origine, celui-ci est généralement assuré par la famille. C'est l'occasion d'utiliser ses réseaux familiaux qui vont accueillir et guider le jeune dans la grande ville, à Kamsar et souvent à la capitale, Conakry.

« Je faisais des études coraniques à Tougnifily... À l'image de mes amis du village j'ai pris l'initiative d'apprendre un métier. Mon père s'était opposé à mon départ mais finalement nous nous sommes compris quand j'ai commencé l'apprentissage de la maçonnerie à côté de ma tante maternelle à Conakry. J'étais apprenti chez un maître qui travaillait dans une société de construction. " Homme, Maçon, Brika, 29 ans

Parfois, l'apprenti loge chez son maître d'apprentissage ; dans ce cas, celui-ci ne réclame pas de contrepartie financière à l'hébergement, étant dédommagé par le travail de l'apprenti.

La durée de l'apprentissage varie donc beaucoup suivant les métiers et la période de la vie pendant laquelle on l'entreprend. Les métiers « traditionnels » sont acquis par un apprentissage très précoce qui s'échelonne sur plusieurs années, les métiers « intermédiaires » sont appris un peu plus tard et sur une durée plus courte, permettant éventuellement à l'enfant de suivre une partie de la scolarité dans le primaire. Quant aux métiers « modernes », l'apprentissage débute plus tard, le plus souvent en ville et dure environ deux ans. Ce dernier parcours est celui qui laisse l'espace de capabilité le plus large, tout d'abord puisqu'il n'entre pas en concurrence dans le temps avec la scolarisation de base, mais aussi parce qu'il permet une plus grande liberté de choix entre les différents fonctionnements envisageables.

\section{Les sorties d'apprentissage}

Un quart des personnes débute son activité chez son maître d'apprentissage, mais pour sept personnes sur 
dix, la formation débouche sur une activité à son propre compte. Plusieurs contraintes conditionnent la sortie de l'apprentissage, comme la constitution du capital de départ ou l'acquisition d'outillage. Ces facteurs de conversion sont liés à la pauvreté de l'environnement économique. Ce sont donc typiquement des contraintes d'accessibilité.

«Je faisais des bricoles pendant l'apprentissage. Ces bricoles m'ont permis de m'acheter certains outils de travail. Mon beau-frère aussi m'avait payé des outils. Ces outils m'ont permis d'ouvrir mon propre atelier de travail. » Homme, Mécanicien, Brika, 33 ans

"Parfois, j'accompagnais ma mère au marché. Un peu plus tard, j'ai pu remplacer ma mère sur le marché de poisson, je partais à sa place. C'est à partir de ce moment que j'ai compris l'intérêt qu'on pouvait tirer du poisson. Maintenant, j'avais besoin d'argent pour m'installer. C'est pourquoi j'ai pris une dette auprès de mon grand frère pour entreprendre le commerce de poisson à mon propre compte. Je négociais du poisson avec les pêcheurs locaux du village, je le fumais avant de le mettre au marché à côté de celui de ma mère. Je faisais à l'image de ma mère. J'ai exercé cette activité jusqu'à la veille de mon mariage. " Femme, Fumeuse de poisson, Kankouf, 27 ans.

L'accès aux savoirs techniques est donc très largement conditionné par la famille (et donc le groupe social), qui décide, au moins en partie, de l'activité que l'individu devra exercer. Les personnes sont cependant très inégales quant à la liberté de choix ; cette inégalité dépend de plusieurs facteurs de conversion : l'appartenance à un groupe social casté ou pas, le sexe de la personne, les opportunités sociales, comme avoir de la famille en ville ou se faire accepter en apprentissage chez un artisan, les opportunités économiques pour démarrer l'activité.

\section{DES DIFFÉRENCES DE STATUT SOCIAL À LA VALORISATION INÉGALE DES MÉTIERS}

Un grand nombre des activités hors agriculture sont pratiquées de manière complémentaire à celle-ci, dans le cadre d'une multiactivité qui a pour fonction de sécuriser le système productif familial. Cependant, certains métiers sont plus valorisés que d'autres, socialement, mais aussi sur le plan financier. Nous comparons ci-dessous les niveaux de revenus du ménage, non comme unique mesure du bien-être, mais aussi comme un indicateur de l'efficience des fonctionnements accomplis à travers la multiactivité, afin d'équilibrer les ressources du ménage.

La base "pauvreté», dont est extraite la base « multiactivité », permet de constituer un groupe témoin de ménages ne pratiquant aucune des activités étudiées ici. Ce groupe témoin est composé de 349 ménages, contre 496 ménages pour le groupe multiactif.

\section{La fonction de sécurisation des revenus}

Dans ce cadre, on vérifie quels effets la pratique d'une ou plusieurs activités en dehors de l'agriculture produit sur les revenus du ménage. Le revenu présenté ci-dessous est le revenu disponible brut par unité de consommation ${ }^{13}$, il est calculé en faisant la somme des revenus monétaires et de la valeur de la production autoconsommée.

Le graphique 1 présente la distribution des ménages en douze classes de revenu en comparant le groupe témoin et le groupe «multiactifs ». On constate pour le groupe « multiactifs » à la fois l'effacement partiel des revenus les plus faibles et l'apparition d'un groupe de ménages aux revenus élevés. Le revenu médian annuel par unité de consommation est de 470000 FG (195 euros) pour les ménages « multiactifs » contre 320000 FG (133 euros) pour le groupe témoin. ${ }^{14}$

Mais il existe de fortes inégalités à l'intérieur du groupe des «multiactifs ». Elles sont liées en partie à la nature des activités exercées, mais aussi aux conditions dans lesquelles celles-ci sont exercées.

\footnotetext{
13 Revenu de l'ensemble des activités ramené à l'unité de consommation qui permet de prendre en compte la taille et la composition du ménage en utilisant l'échelle d'équivalence d'Oxford: le premier adulte compte pour 1 , les suivants pour 0,7 , les enfants de moins de 15 ans pour 0,5 .

14 En juin 2004,1 euro $=2400$ Francs Guinéens. Un an plus tard (mi 2005), 1 euro $=4260$ Francs Guinéens. Cette chute semble enrayée mi 2006, avec un cours autour de 1 euro pour $5500 \mathrm{FG}$.
} 


\section{Graphique 1}

\section{Comparaison de la distribution des revenus selon la nature des activités du ménage}

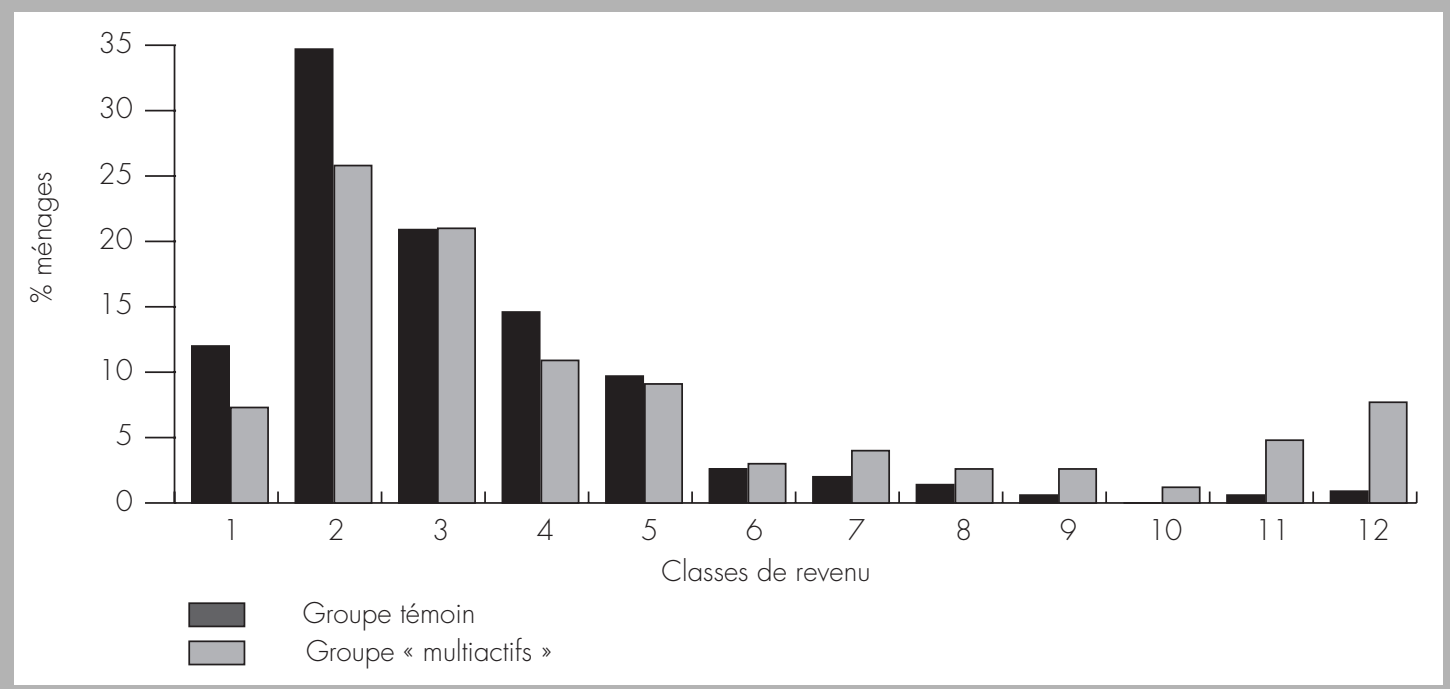

Source : nos propres calculs.

- groupe témoin (ménage dont aucune personne ne pratique une des activités sélectionnées) : 349 ménages (41\%).

- groupe « multiactifs » (ménages dont au moins une personne pratique une des activités sélectionnées) : 496 ménages (59 \%).

- Revenu : Revenu disponible brut par unité de consommation (monétaire et autoconsommation valorisée). Classe de revenu de $150000 \mathrm{FG}$; exemple de lecture : la classe 1 dispose d'un revenu de $150000 \mathrm{~F}$ par unité de consommation ; la classe 2 de 150000 à 300000 ; la classe 12 quant à elle dispose d'un revenu supérieur à $150000 \mathrm{FG}$ x 11 .

\section{Des métiers à l'attractivité financière disparate}

D’une analyse plus fine des revenus des ménages pratiquant la multiactivité, il ressort de fortes disparités dans l'attractivité financière de ces métiers ${ }^{15}$ (graphique 2). Quatre groupes de métiers ont été distingués: les métiers castés (forgeron, potière), les métiers intermédiaires (activités du bâtiment, menuiserie, couture, artisanat alimentaire), les métiers modernes (réparateurs, mécaniciens, chauffeurs, photographe) et les pêcheurs.

Le graphique 2 révèle que les ménages auxquels appartiennent les forgerons et potières sont concentrés dans les classes de revenus les plus bas. Les activités «intermédiaires », caractérisées par le fait que l'apprentissage ne nécessite pas forcément un séjour en ville, sont plus attractives. Les revenus provenant des activités «modernes», se révèlent fragiles en

\footnotetext{
15 On pourra s'étonner de la faiblesse des revenus ; mais ce serait oublier que le contexte de l'étude est celui de la pauvreté rurale dans un pays africain.
}

milieu rural, en raison notamment d'une faible demande solvable. Le groupe dominant les classes de revenu élevés est celui des pêcheurs, et plus particulièrement les pêcheurs en activité principale. Ils tirent le plus de profit de l'insertion dans les circuits économiques marchands.

\section{Un espace de capabilités réduit pour les métiers castés}

Il peut sembler surprenant que des métiers qui exigent une forte technicité et un long apprentissage soient aussi les plus mal valorisés. La faiblesse des revenus de ces ménages s'explique par deux facteurs. Le premier est le déclin du rôle des forgerons dans les collectivités villageoises depuis plusieurs décennies. Les trois domaines qui leur étaient dévolus sont affectés : sur le plan technique, avec l'abandon de la fonte du minerai, sur le plan cérémoniel par le recours plus fréquent aux centres de santé pour la circoncision et sur le plan religieux du fait de 


\section{Graphique 2}

\section{Attractivité financière de quatre groupes d'activité (principale et secondaire)}

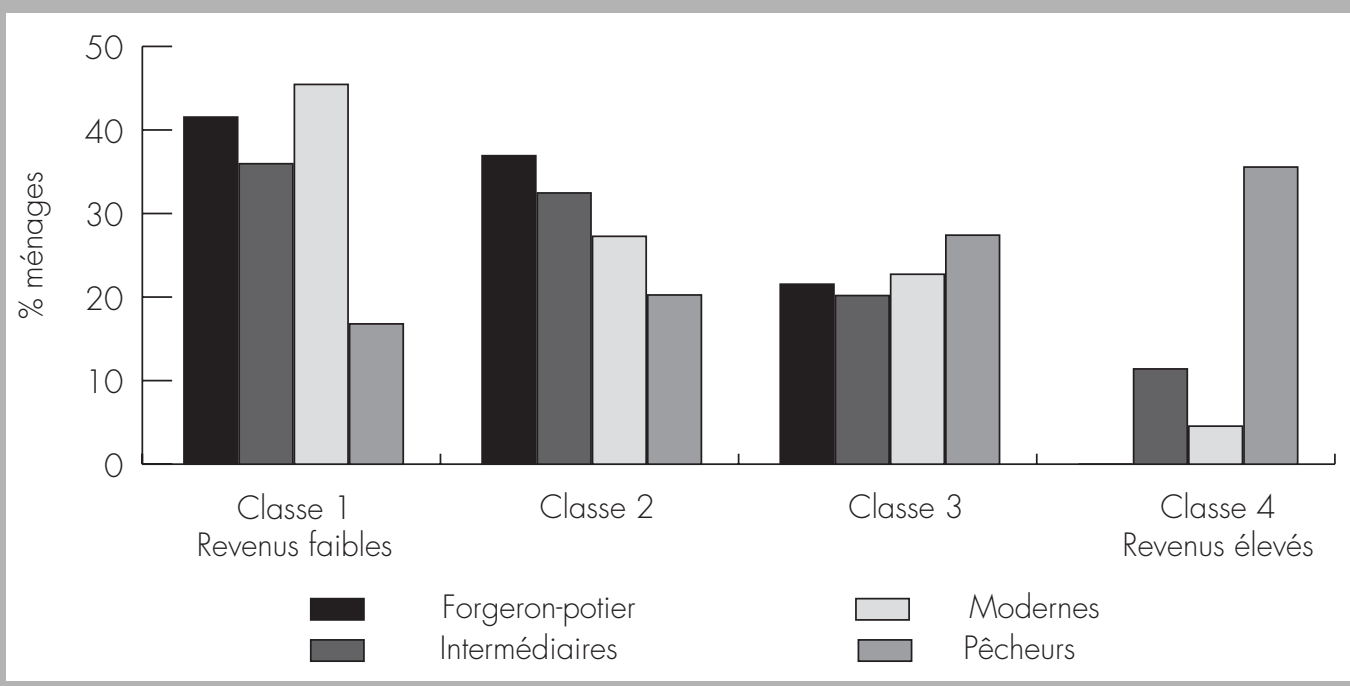

Source : nos propres calculs.

Revenus divisés en quartile de revenu annuel disponible brut par unité de consommation en 2004 :

- classe $1:$ revenus faibles : $<100 €$

- classe $2:$ de 100 à $200 €$

- classe $3:$ de 200 à $460 €$

- classe 4 revenus élevés : plus de $460 €$

Exemple de lecture : $40 \%$ des ménages de forgerons-potiers mais aussi $45 \%$ des ménages où une personne exerce un métier « moderne » ont un revenu inférieur à $100 €$ par unité de consommation et par an.

l'islamisation qui réduit le recours à des pratiques animistes. Le second est plus général: bien que disposant par leur travail d'un prestige important, ils appartiennent à un milieu social inférieur; cette situation paradoxale a été analysée par Meillassoux : «détenteurs de cet instrument de puissance (le savoir) ces "sages" sont aussi l'objet de mesures visant à les neutraliser politiquement. Ils seront soit recrutés dans un milieu social inférieur, soit castés. » (Meillassoux, 1960)

C'est ce qui se passe là où l'islamisation et la stratification sociale sont les plus prononcées : la situation des forgerons y est économiquement très précaire. Alors que l'apprentissage s'effectue dès l'enfance, empêchant la scolarisation, l'acquisition des savoirs techniques ne sert qu'à engager les individus dans un métier défavorisé et à les fixer dans une situation sociale inférieure. Parce que leur espace de capabilités et leur liberté "à faire et à être » sont très réduits, ce processus les maintient dans un cercle de reproduction de la pauvreté. Pour en sortir, il ne leur reste que la possibilité d'émigrer vers la ville.

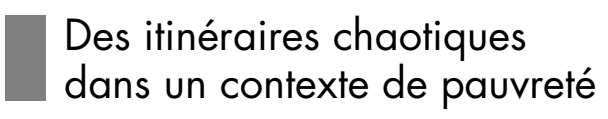

En dehors des spécificités des métiers castés, la pratique des activités des autres métiers rencontre aussi un certain nombre de problèmes, plutôt liés au sous-équipement en services et à la pauvreté persistante. Ainsi, il est difficile de rassembler un capital pour démarrer l'activité dans un milieu où les institutions financières sont encore peu implantées ${ }^{16}$, aussi la famille est-elle sollicitée pour aider au démarrage. Ensuite, les activités sont très sensibles à une conjoncture qui, depuis des années, se dégrade : la faiblesse de la demande solvable, la dépréciation continue de la monnaie nationale, l'absence de

16 À l'exception du Crédit Rural de Guinée, qui est une institution de micro-finance. 
confiance dans des institutions corrompues contribuent à maintenir une grande précarité des activités économiques. Les récits de vie recueillis illustrent bien ces problèmes :

«Le début n'était pas facile, mais progressivement l'habileté est venue. Il fallait augmenter la longueur du filet, j'ai alors emprunté de l'argent au crédit rural de Mankountan. Ce qui m'a permis d'avoir un nouveau filet. Parfois je négocie avec certains usuriers pour avoir le filet et je les rembourse en poisson. Je fais la riziculture pendant la saison des pluies et la maçonnerie pendant la saison sèche. La pêche est périodique. » Pêcheur, Bigori, 44 ans.

Le matériel est difficilement renouvelé en raison de la faiblesse des marges ou des difficultés de gestion. D'autre part, l'enclavement du milieu rural affecte et rend irrégulier l'approvisionnement et impose des contraintes fortes. Ce phénomène est aggravé par la forte dépréciation monétaire qui existe depuis 2003.

"Le seul problème que nous rencontrons dans cette activité est l'accroissement démesuré des prix sur le marché. Le prix du haricot monte tous les jours. L'huile de palmiste est parfois difficile à avoir. D'ailleurs, il y a deux semaines, je ne suis pas parvenue à l'avoir à Tougnifily" Vendeuse de beignets, Brika, 25 ans

Les débouchés commerciaux restent très étroits, en raison d'une demande faible, ou d'une solvabilité limitée sur les marchés locaux.

«À Bigori, je n'ai pas bénéficié de grands contrats de travail, c'est pourquoi je pratique l'agriculture parallèlement à la menuiserie. Les habitants du village n'accordent pas beaucoup d'importance à ce métier, ils n'achètent pas de meubles » Menuisier, Bigori, 46 ans.

Mais aussi, dans un contexte social où l'activité économique marchande est mal dégagée d'un échange de services entre parents ou voisins, il est difficile de rentabiliser l'activité.

« À Brika, j'avais ouvert mon atelier de menuiserie, finalement j'ai compris que ce métier ne serait pas très bénéfique pour moi au village où je suis né car les parents ne payaient pas les prestations. C'est pourquoi j'ai abandonné l'exercice de ce métier au village pour retourner à Kamsar » Réparateur radio, Brika, 37 ans.

À l'inverse, les pêcheurs qui ont pu acquérir du matériel moderne (filet et pirogue motorisée) sont intégrés par une filière commerciale efficace, au marché urbain de Conakry et même de Dakar. Les contraintes d'accès sont moins sociales qu'économiques ou personnelles, comme la capacité à acheter du matériel ou le savoir-faire dans le métier.

Les facteurs de conversion présentent des contraintes spécifiques selon les groupes sociaux ou selon le sexe et conditionnent donc le type de métier et la formation auxquels peut prétendre une personne. En outre, les débouchés locaux sont très réduits en raison de la pauvreté monétaire des ménages et seuls ceux qui ont pu s'insérer dans une activité correspondant à une forte demande urbaine, celle du poisson de mer, parviennent à dégager des revenus conséquents. Enfin, la déficience des services publics et la faiblesse de l'armature socio-spatiale du territoire engendrent aussi une grande insécurité dans la conduite des activités. La valorisation économique et sociale des métiers de la multiactivité est donc inégale.

\section{L'ÉDUCATION, POUR AGRANDIR L'ESPACE DES CHOIX?}

A. Sen a montré, par de nombreux exemples, que l'éducation permettait à l'individu d'élargir le champ de ses libertés en lui permettant d'acquérir une relative autonomie et de mieux participer au débat public (Sen, 2000). Cela est particulièrement vrai pour les femmes, pour qui des inégalités de sexe se superposent aux inégalités sociales : l'accès à l'éducation desserre la contrainte sociale en favorisant une ouverture de l'espace des choix, en plus d'être supposée favoriser l'amélioration de la productivité du travail. Les conséquences positives dans de nombreux domaines économiques ou sociaux ont été soulignées : amélioration de l'autonomie et des revenus des femmes, meilleur contrôle des naissances, amélioration de l'hygiène dans les soins aux enfants entraînant une diminution de la mortalité infantile. 
Cependant, en Guinée, et plus particulièrement en Guinée Maritime, l'offre en matière d'enseignement technique et professionnelle est extrêmement réduite. L'enseignement technique et professionnel comprend aussi les écoles d'instituteurs, l'école de sagesfemmes, l'école d'infirmiers, la formation à l'hôtellerie, etc., métiers qui nous intéressent moins directement dans cette étude. Les quelques centres de formation professionnelle (construction, électricité, couture) sont presque tous situés à Conakry, la capitale. Ils ont formé, en 2004, 700 personnes. La population de la Guinée était estimée à l'époque à 9,5 millions d'habitants, la moitié de la population ayant moins de 18 ans. De même, pour accéder à cet enseignement, la condition préalable est d'avoir achevé un cycle complet dans l'école primaire.

\section{Un faible niveau} d'éducation de base

Le niveau de formation de la population adulte de Guinée Maritime est très bas, avec un important écart entre hommes et femmes. Les femmes des ménages pratiquant la multiactivité ont à peu près les mêmes caractéristiques que celles des ménages ne la pratiquant pas, avec un niveau de formation qui est extrêmement faible : $8 \%$ des femmes adultes du groupe « multiactifs » ont été à l'école contre $6 \%$ du groupe témoin et dans les deux cas, moins de $3 \%$ d'entre elles ont achevé le cycle primaire (Droy, 2005).

Du côté des hommes, les différences entre les groupes sont un peu plus marquées. Les hommes des ménages « multiactifs » sont un peu moins nombreux à avoir été à l'école et à avoir achevé le primaire (10\% contre $15 \%)$. Ils ont donc une formation de base de type formel un peu plus faible que ceux du groupe témoin.

Mais le cas des pêcheurs en activité principale, qui ont des revenus nettement plus élevés que la moyenne, est particulièrement intéressant. Ont-ils pu profiter du levier que constitue l'éducation pour s'engager dans cette activité particulièrement rentable? La comparaison des niveaux de formation de base entre les pêcheurs (exclusivement des hommes) et les autres hommes pratiquant la multiactivité ne montre pas de différences significatives. Ce sont donc, dans ce cas précis, d'autres opportunités sociales qui ont été déterminantes pour le choix des activités ${ }^{17}$.

En revanche, la dynamique actuelle montre une forte hausse des taux de scolarisation dans le primaire, avec des inégalités de sexe qui tendent à se réduire. En outre, le fait qu'un adulte du ménage ait été scolarisé a une incidence positive sur le choix de scolarisation des enfants et plus particulièrement des filles. Il n'est pas possible actuellement de mesurer l'impact de la scolarisation sur la situation économique des ménages ruraux ; cependant, ces derniers perçoivent bien l'intérêt de la scolarisation pour le futur de leurs enfants.

\section{Pouvoir aller et pouvoir rester à l'école}

Les profondes inégalités spatiales dans les niveaux de scolarisation s'expliquent en partie par la disponibilité ou non de l'offre scolaire à proximité des lieux de résidence. Cette offre tend cependant à augmenter ces dernières années, la Guinée étant engagée dans le programme « Éducation pour Tous ».

Mais l'accès à l'école ou l'arrêt précoce des études est aussi entravé par la faiblesse du niveau de vie. Chez les adultes, la principale raison d'abandon des études est économique : manque de moyens financiers, nécessité de trouver une activité rémunérée ou besoin de main-d'œuvre sur les champs familiaux. Ces motifs sont invoqués par $55 \%$ des hommes du groupe «multiactifs» contre $37 \%$ de ceux du groupe témoin.

Pour les femmes ${ }^{18}$, ces difficultés sont aussi mentionnées $(20 \%)$. Mais à ces entraves économiques s'ajoutent, pour les femmes, des raisons sociales: mariages précoces et grossesses sont des motifs fréquents d'arrêt des études (37\%). Une étude réalisée en 1998 sur la Guinée montre le poids déterminant de la représentation de la place des femmes

\footnotetext{
17 L'échantillon ne comporte que des personnes adultes du milieu rural, et qui donc y sont restées ou sont revenues. Nous n'avons pas ici d'information sur la mobilité géographique et sociale que peut procurer l'accès à l'éducation, surtout secondaire et supérieure.

${ }^{18}$ En raison du faible nombre de femmes ayant été à l'école aussi bien dans le groupe témoin que dans le groupe «multiactifs», nous n'avons pas fait la distinction entre ces deux groupes pour l'analyse des motifs d'arrêt des études.
} 
dans la société pour expliquer les inégalités de sexe dans l'accès à l'école (Colclough et al., 1998), représentation qui a les mêmes répercussions sur les choix d'accès aux métiers.

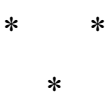

On considère en général que toute formation ou acquisition de savoirs enrichit le capital humain de l'individu et alimente le progrès collectif. Et, effectivement, dans les villages de Guinée Maritime, l'usage des savoirs techniques, sous la forme d'une activité principale ou secondaire, permet à la plupart des ménages d'atténuer les irrégularités de la production agricole et d'alléger la soudure, lorsque les récoltes ont été insuffisantes.

Cependant, une analyse plus détaillée de l'accès aux formations met en évidence les difficultés de conversion des libertés formelles en libertés réelles dans l'accès aux savoirs, ce qui conditionne ensuite l'accès à des métiers plus ou moins valorisants et rémunérateurs. Les différences économiques révèlent ainsi deux sortes de savoirs techniques. Des savoirs modernes, dont l'apprentissage, même s'il peut être parfois long, difficile ou nécessiter le déplacement hors du village, est ouvert aux hommes appartenant aux groupes sociaux les moins défavorisés. La valorisation de ces savoirs sous la forme d'un métier exercé au village, même si elle ne permet ici qu'une insertion malaisée dans les circuits économiques nouveaux, dépendra de conditions communes à tous. À l'opposé, certains savoirs traditionnels sont d'une autre nature. Leur accès est lié au sexe ou à l'appartenance à un groupe. Ils sont enchâssés dans la culture, intègrent des normes sociales et, loin de permettre aux individus d'améliorer leur condition, ils reproduisent les inégalités auxquelles ils participent: statut mineurs des cadets, inégalités de sexe, infériorité de castes. L'apprentissage non formel au sein des structures familiales présente sans doute des avantages pour la qualité de la transmission de savoir-faire, mais contribue aussi à la transmission intergénérationnelle des inégalités sociales.

\section{Bibliographie}

Bouju S. (1999), «Le morcellement identitaire des populations littorales. Quelques éléments de l'histoire du peuplement », in Dynamiques et usages de la mangrove dans les rivières $d u$ sud. Cormier-Salem Éd., IRD, pp. 131-138.

Boutrais J. (1999), «Pasteurs du Sud, Pasteurs du Nord ... et les autres », in Le pouvoir du savoir de l'Arctique aux Tropiques, Holtedahl et al. Ed., Karthala, pp. 21-40

Colclough C., Rose P., Tembon M. (1998), Gender inequalities in primary schooling: the roles of poverty and adverse cultural practice, IDS working paper 78, $31 \mathrm{p}$.
Droy I. (2005), «Pauvreté, enclavement et scolarisation en milieu rural guinéen ", Mondes en développement, vol. 33-2005/4, n 132, "Éducation et développement ", pp. 111-127.

Droy I. (2006), «Quel apport de l'approche par les capabilités pour l'analyse des inégalités de sexe? Amartya Sen, un économiste du développement», V. Reboud Ed., AFD, Notes et documents, $\mathrm{n}^{\circ} 30$.

Dubois J.-L., Mahieu R., Poussard A., (2001) «La durabilité sociale comme composante d'un développement humain durable», in Le développement humain, Cahiers du Gratice, Université de Paris XII - Val de Marne, pp. 95-133. 
Guerin I., (2003), Femmes et économie solidaire, La Découverte, MAUSS SED.

Kyburz O. (1997), «La fabrication de la foulanité », Journal des africanistes, 67(2), pp. 101-126.

Meillassoux C. (1960), «Essai d'interprétation du phénomène économique dans les sociétés traditionnelles d'autosubsistance », Cahier d'Études Africaines, ${ }^{\circ} 4$, pp. 38-66.

Meillassoux C. (1975), Femmes, greniers et capitaux, Maspéro, Paris, 217 p.

Marie A. (Ed.) (1997), L'Afrique des individus, Karthala, Paris, 438 p.

Nussbaum M. (2000), Women and Human Development. The capabilities approach. Cambridge University Press, Cambridge.

Person Y. (1968), Samori - Une révolution dyula, Mémoires de l'Institut Fondamental d'Afrique Noire, $\mathrm{n}^{\circ} 80$, Dakar, $2377 \mathrm{p}$.

Poirot J. (2005), «Le rôle de l'éducation dans le développement chez J. Rawls et A. Sen, entre équité et efficacité », Mondes en développement, vol. 33-
2005/4, n¹32, "Éducation et développement 》, pp. 29-38.

Robeyns I. (2005), "Capability approach, a theoritical survey", Journal of human development, vol. 6, $\mathrm{n}^{\circ} 1$.

Rossi G., Bazzo D., Lauffer M. (2002), « La Guinée Maritime aujourd'hui», Les cahiers d'outre-mer, $\mathrm{n}^{\circ} 217$, pp. 31-62.

Sen A.K. (1987), Commodities and Capabilities, Oxford India Paperbacks, Oxford University Press, Oxford, $89 \mathrm{p}$.

Sen A.K. (1992), Inequality Reexamined, Oxford University Press, Oxford, 207 p.

Sen A.K. (1993), Capability and Well-Being. The Quality of Life, M.C. Nussbaum and A. Sen (Eds) Clarendon Press, Oxford, pp. 30-53.

Sen A.K. (2000), Un nouveau modèle économique: développement, justice, liberté, Odile Jacob.

Silber J. (2001), «Amartya Sen et la mesure de la croissance économique et du développement social», Revue d'économie $d u$ développement, 3/2001, pp. 107-135.

\title{
Résumé
}

\section{Les inégalités d'accès aux savoirs techniques en Guinée Maritime}

\author{
Jean-Étienne Bidou et Isabelle Droy
}

En milieu rural guinéen (Guinée Conakry) comme dans la plupart des pays en développement, la transmission des savoirs techniques s'effectue essentiellement dans un cadre familial, parfois comme apprentis auprès d'artisans, et donc pas dans le cadre d'un enseignement technique organisé et formalisé. L'accès à ces modes d'apprentissage est soumis à des règles encore mal décryptées. Si la formation et la pratique de certaines activités sont liées à l'appartenance à un groupe social particulier, d'autres métiers, correspondant à de nouvelles activités, semblent plus ouverts. Une approche sous l'angle des capacités, issue des travaux d'A. Sen, permet de distinguer les libertés réelles d'accès aux apprentissages en fonction des opportunités sociales: appartenance à un groupe ou une ethnie, selon le sexe ou en fonction des réseaux familiaux. Ainsi, certains métiers apparaissent plus valorisés socialement et économiquement que d'autres et les contraintes d'accès aux savoirs techniques perpétuent les inégalités de capabilités et entretiennent la vulnérabilité de certains groupes sociaux.

\section{Mots-clés}

Approche par les capabilités, processus d'apprentissage, savoir professionnel, inégalités, Guinée Journal of Economic Literature: D 63, J 24, O 18, 121 


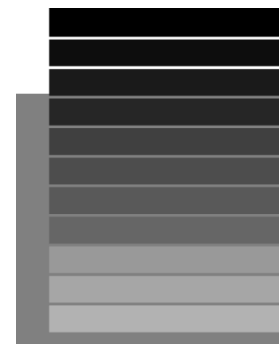

revue

semestrielle
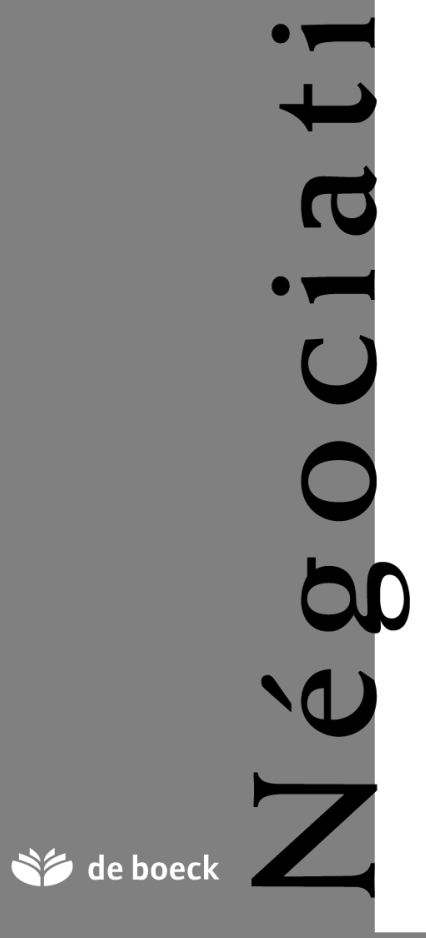

\section{N॰7 - Printemps 2007}

\section{Varia}

Jean-Marc Siroën

L'OMC et les nédociations commerciales multilatérales

Benoît Bernard

L'impact d'un indicateur de gestion dans la négociation sylvicole

Patrick Germain-Thomas

La coopération culturelle intercommunale

Jeanne Duvallet, Alexis Garapin et Daniel Llerena

Coordination par la nédociation

Elisabeth Volckrick

Intervenir en tiers aujourd'bui

\section{Forum}

Laurence de Carlo

Les différentes pbases du vocabulaire de la nédociation

Noelle Aarts et Cees van Woerkum

Rbétorique et réalités des délibérations publiques

Grand Entretien

Rencontre avec Robert McKersie

"Qu'est-ce qui est le plus difficile, la négociation

à la table principale...? "

Bonnes feuilles

Richard Walton et Robert McKersie

Négociations sociales en relations internationales

et pour les droits cividues

\section{Pédagogie / Formation}

Jean-Guy Bergeron, Reynald Bourque

et Franck White

Évaluation d'un prodramme de formation

Notes de lecture/ (Re)lectures

$$
\begin{gathered}
\text { Au sommaire du n }{ }^{\circ} 5 \text { : } \\
\text { Philip Milburn, Hubert Touzard, Sanda Kaufman, } \\
\text { Lavinia Hall, Jacques Faget, Marwa Daoudy, } \\
\text { Isabelle Leroux, Louis Simard, Alice Le Flanchec, Arnaud Stimec, } \\
\text { Rencontre avec Tony Vandeputte } \\
\text { Négociations est disponible en texte intégral } \\
\text { sur www.CAIRN.INFO }
\end{gathered}
$$

Négociations. Une nouvelle revue en langue française, dont l'ambition est $d^{\prime}$ instruire la question de la négociation dans la diversité de ses dimensions, en favorisant la confrontation interdisciplinaire et en faisant se rejoindre plusieurs traditions d'étude.

Éditorial et conditions d'abonnement sur le site de la revue :

\section{http://universite.deboeck.com/revues/negociations/}

Négociations. CRIS, Département de sciences sociales.

Faculté d'Économie, de gestion et de sciences sociales, bd du Rectorat, 7, B-4000 Liège

Directeurs de la publication : Olgierd KUTY (Université de Liège), Christian THUDEROZ (INSA de Lyon) 